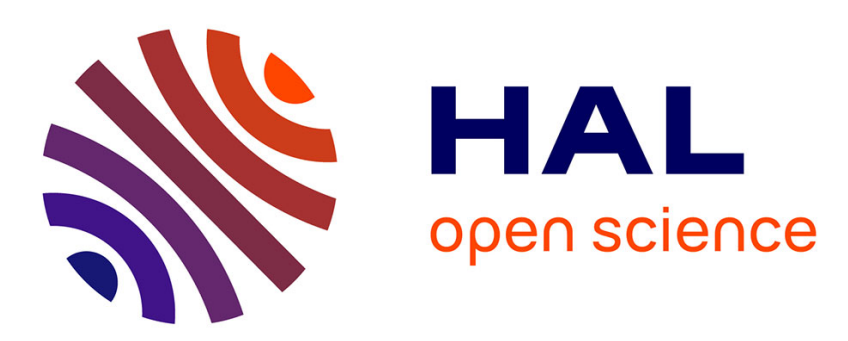

\title{
Practical methods to reduce impurities for gram-scale amounts of acidic sophorolipid biosurfactants
} Niki Baccile, Anne-Sophie Cuvier, Claire Valotteau, Inge van Bogaert

\section{To cite this version:}

Niki Baccile, Anne-Sophie Cuvier, Claire Valotteau, Inge van Bogaert. Practical methods to reduce impurities for gram-scale amounts of acidic sophorolipid biosurfactants. European Journal of Lipid Science and Technology, 2013, 115 (12), pp.1404-1412 10.1002/ejlt.201300131 . hal-01455116

\section{HAL Id: hal-01455116 https://hal.sorbonne-universite.fr/hal-01455116}

Submitted on 3 Feb 2017

HAL is a multi-disciplinary open access archive for the deposit and dissemination of scientific research documents, whether they are published or not. The documents may come from teaching and research institutions in France or abroad, or from public or private research centers.
L'archive ouverte pluridisciplinaire HAL, est destinée au dépôt et à la diffusion de documents scientifiques de niveau recherche, publiés ou non, émanant des établissements d'enseignement et de recherche français ou étrangers, des laboratoires publics ou privés. 
IMPORTANT NOTE : Please be aware that slight modifications occurring after Proof correction may occur between this version of the manuscript and the version on the Publisher's website-

\title{
Practical methods to reduce impurities for gram-scale amounts of acidic sophorolipid biosurfactants
}

\section{Authors}

Niki Baccile, ${ }^{\mathrm{a}, \mathrm{b}, \mathrm{c} *}$ Anne-Sophie Cuvier, ${ }^{\mathrm{a}, \mathrm{b}, \mathrm{c}}$ Claire Valotteau, ${ }^{\mathrm{a}, \mathrm{b}, \mathrm{c}}$ Inge N. A. Van Bogaert ${ }^{\mathrm{d}}$

\author{
Affiliations \\ a-UPMC Univ Paris 06, UMR 7574, Chimie de la Matière Condensée de Paris, F-75005, \\ Paris, France. E-mail : niki.baccile@upmc.fr \\ b- CNRS, UMR 7574, Chimie de la Matière Condensée de Paris, F-75005, Paris, France \\ c - Collège de France, UMR 7574, Chimie de la Matière Condensée de Paris, F-75005, Paris, \\ France \\ d. InBio, Department of Biochemical and Microbial Technology, Faculty of Bioscience \\ Engineering, Ghent University, Coupure Links 653, 9000, Ghent, Belgium
}

\begin{abstract}
Raw sophorolipids, a variety of bioderived glycolipid biosurfactants produced by the yeast Candida bombicola, generally present themselves as a mixture of acetylated acidic and lactonic forms mixed with residual fatty acids. To recover the acidic form alone in large amounts, e.g. tens of grams, which is interesting for various applications in cleaning technologies, material science, medicine, one must either purify the mixture through column chromatography, thus tremendously reducing the final yield, or remove the fatty acids via hot hexane extraction followed by alkaline hydrolysis and a liquid-liquid extraction process; in the latter, pentanol, used as extraction medium, is a high boiling point solvent and, consequently, difficult to remove under standard vacuum extraction conditions. Here, we compare, quantify and draw the positive and negative aspects of the typical hexane/pentanol extraction route compared to two additional ones, never reported for this system: a cold extraction from a pentanol/alcane mixture and a consecutive filtration on inverse C18modified silica phase and silica gel in, respectively, water/acetonitrile and methanol/ $\mathrm{CH}_{2} \mathrm{Cl}_{2}$ mixtures. The first one generates highly pure products but quantitative extraction is quite long
\end{abstract}


(up to several days) while the second is an extremely handy and rapid (few hours) way but it is less quantitative and it provides a product with not less than $6 \%$ impurities.

\section{Introduction}

The emerging field of bio-based surfactants has been intensively studied in the past 20 years $^{1}$ in agreement with the 12 principles of green chemistry. Glycolipids are surface active agents composed of a hydrophilic carbohydrate-based head linked to a fatty acid or a fatty alcohol through an ester, an amide or an ether bond. ${ }^{2}$ They already replace, and/or are destined to massively replace, classical surfactant families like alkylsulfates or alkylammonium in home and personal care products. For these reasons, their physicochemical properties have been studied in the past 15 years. Effect of molecular structure (e.g., chain length, number of sugar units), concentration, use of co-solvents (short-chain alcohol, for instance), co-surfactants on the critical micelle concentration $(\mathrm{cmc})$, surface tension, rheology, cleaning power but also self-assembly and mesophase formation have shown particular perspectives for many of these compounds..$^{2-4}$ These molecules are generally synthesized via several chemical strategies, reviewed by von Rybinski, from renewable compounds like fatty acids and sugars. In term of their cost, they are still more expensive in their pure form with respect to classical surfactants. For instance, commercial alkylpolyglucosides (APG) are actually sold as formulations presenting either a non-constant number of glucose units or mixtures of at least two different chain lengths.

In this context, various authors have shown the interest of a specific class of biosurfactants, sophorolipids (Figure 1), a family of entirely bio-derived glycolipids. These natural compounds can be obtained in large amounts by yeast culture in presence of several carbon sources, like glucose, fatty acids but even alkanes and waxes. ${ }^{5-8}$ Due to their reduced environmental impact biosynthesis, confirmed by a recent life cycle analysis study, ${ }^{9}$ sophorolipids (SL) have attracted a fair attention in various fields: skin-care industry, ${ }^{10-12}$ anticancer, $^{13}$ structure-directing agents and self-assembly properties. ${ }^{8,14,15,16,17}$ Raw sophorolipids are a mixture of two forms, lactonic and acidic, having different degrees of acetylation. Nevertheless, the acidic non-acetylated form of sophorolipids has gained much interest and has been exploited in most of the above-cited studies. For this reason, it becomes quite important to obtain acidic sophorolipids (SL-COOH) from the raw mixture. Unfortunately, at the moment, the development of a full microbial approach to accomplish such a task is still a matter of ongoing studies in biotechnology-specific laboratories. A modified strain producing non-acetylated sophorolipids has been created, but the synthesized 
molecules are still a mixture of acidic and lactonic forms and yield is much as less as compared to the wild type. ${ }^{18}$ Recently, a strain producing solely acidic sophorolipids was constructed and attempts to combine both features in one strain are still ongoing. The common strategy to obtain non-acetylated acidic sophorolipids exists in the application of chemical conversion and purification procedures to the raw mixture of sophorolipids, which mainly consists into: 1) hexane washing to remove residual fatty acids; 2) alkaline hydrolysis at about $100^{\circ} \mathrm{C}$ followed by acidification; these steps remove all ester groups and are responsible for the production of acetic acid and salt; 3) 1-pentanol extraction, which allows the recover of the SL-COOH, leaving salts and acetic acid in the water phase; 4) silica column purification, typically using methanol/dichloromethane as eluent.

This procedure, even if efficient in obtaining a pure acidic SL-COOH compound, has many drawbacks, listed hereafter. 1) Alkaline hydrolysis can lead to parasite reactions that add impurities to the final compound; 2) 1-pentanol extraction is a tedious step because of its high boiling point (137-139 $\left.{ }^{\circ} \mathrm{C}\right)$, making its removal under vacuum a delicate, potentially time-consuming, task, not exempt of parasite esterification reactions between pentanol itself and the $\mathrm{COOH}$ group of SL-COOH; 3) silica column purification is a complicated operation for large amounts of product and require good practical knowledge in organic chemistry. Significant loss of product can be experienced on the column due to strong attractions between silanols and sugars.

For these reasons, we propose in this work to review the purification of acidic sophorolipids. We compare the classical "pentanol route", for which we provide more experimental details hard to find in the current litterature, to two alternative ones never proposed before for this system: a cold precipitation, referred to a as a "modified pentanol extraction”, and a “double silica filtration”, both more adapted to work with large amounts of product. In the latest method, hydrophilic and hydrophobic impurities are retained on, respectively, chromatographic $\mathrm{SiO}_{2}$ and inversed-SiO $2\left(\mathrm{SiO}_{2}-\mathrm{C}_{18}\right)$ substrates and for which no pentanol is used at all. Finally, we compare the advantages and inconvenients for each route, also providing the final yields. In the end, the goal of this work is to provide the opportunity to choose between more than one route so to optimize effort, experimental time, yields and level of impurities. Further development of any of these routes could eventually be of interest to develop a scale-up purification process.

\section{Experimental}


Sophorolipids mixture was obtained from Sopholiance (France). This product is certified with 54.6 w\% of dry matter, estimated by Mass Spectroscopy and where about 10 $\mathrm{w} \%$ of the dry compound is constituted by residual fatty acids, indicating that the sophorolipid mixture constitutes a little less than $50 \mathrm{w} \%$ of the overall product.

${ }^{1} \mathrm{H}$ solution NMR spectra were acquired on a Bruker AV300 Ultrashielded@ Avance spectrometer equipped with a $5 \mathrm{~mm}(1 \mathrm{H} / \mathrm{BBF}) \mathrm{BBO}$ probe with Z-axis gradient using a standard $30^{\circ}$ flip angle.

HPLC analysis was performed on a Varian Prostar HPLC system using a Chromolith Performance RP-18e 100-4.6mm column from Merck KGaA at $30^{\circ} \mathrm{C}$ and Evaporative Light Scattering Detection (Alltech). A gradient of two eluents, a $0.5 \%$ acetic acid aqueous solution and acetonitrile, had to be used to separate the components. The gradient started at $5 \%$ acetonitrile and linearly increased till 95\% in $40 \mathrm{~min}$. The mixture was held this way for 10 min and was then brought back to $5 \%$ acetonitrile in $5 \mathrm{~min}$. A flow rate of $1 \mathrm{~mL} / \mathrm{min}$ was applied.

Solvent extraction was done on a Büchi Rotavapor ${ }^{\circledR}$ R-210/R-215, equipped with a digital controller V-850, heating bath B-491, vacuum pump V-700 and closed-circuit chiller F-105. Silica gel $60(0.015-0.040 \mathrm{~mm})$ for column chromatography was obtained from Merck and C18-grafted silica powder 40-60 $\mu \mathrm{m}$ (Vrac-C18-500) was obtained from AIT France.

\section{Results and discussion}

The complexity of a sophorolipid mixture is well-known and, at the moment, impossible to avoid. This is merely a result of the expected variation one tends to observe in (complex/nonspecific) biosynthetic systems. A typical mixture can contain various impurities (mainly residual fatty acids) but it is also characterized by a mixture of different sophorolipids with slightly different chain lengths (C16, C18) and saturated/unsaturated double bonds. By far, the most abundant species (60 \% - $80 \%$, according to the origin), which we refer to as SL, is the C18:1, shown in Figure 1. All purification steps performed in this work are not meant in any way to separate sophorolipids molecules with different structural features but only nonSL impurities, which will be detailed below. 


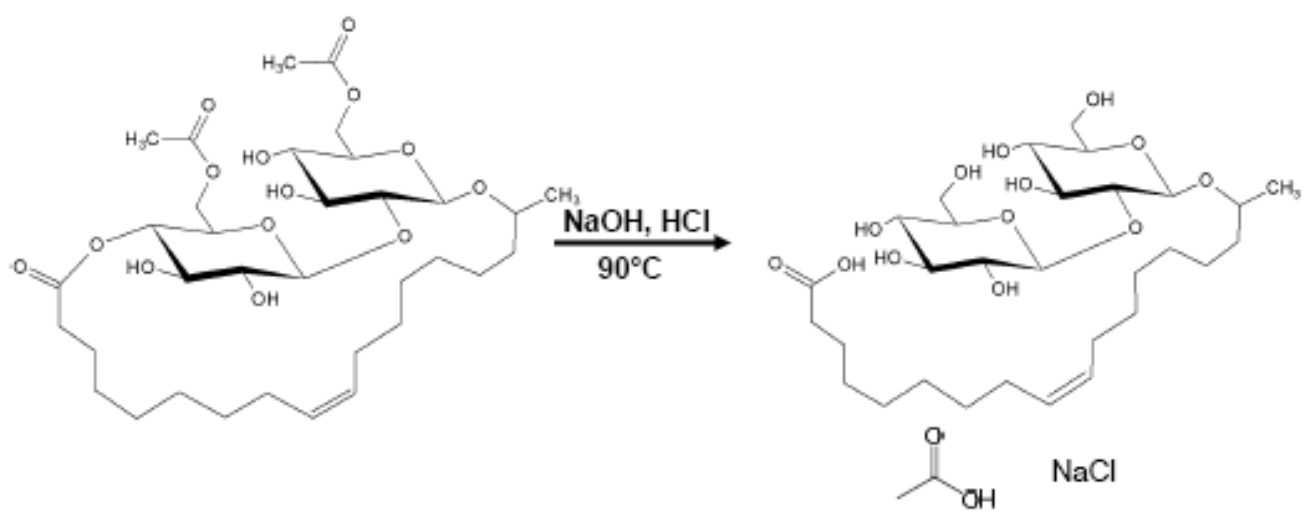

Figure 1 - Alkaline hydrolysis reaction transforming the acetylated lactonic sophorolipid to the deacetylated acidic form of sophorolipids.

Alcaline hydrolysis. The most important step to recover acidic sophorolipids from the raw mixture is the alcaline hydrolysis under heat. Typically, $20 \mathrm{~g}$ of the as-provided Sopholiance mixture is diluted in $50 \mathrm{~mL}$ of a $5 \mathrm{M} \mathrm{NaOH}$ solution and put to reflux under stirring. As for the temperature, we advise to gently increase it up to $90{ }^{\circ} \mathrm{C}$, at which one can leave the solution for $10 \mathrm{~min}$ and then let it cool down to room temperature. The solution should be yellow/pale-yellow from beginning to end. An important risk to obtain side reactions exist if temperatures go beyond $100{ }^{\circ} \mathrm{C}$ due to improper manipulation. This can turn the colour of the solution into dark brown/black, indicating the formation of small amounts of oxidized species. Acidification to $\mathrm{pH}=4$ to recover the SL-COOH is achieved by adding about $40 \mathrm{~mL}( \pm 2 \mathrm{~mL})$ of a $18.5 \mathrm{w} \%$ solution of $\mathrm{HCl}$ under stirring to the cooled mixture. Figure 2 shows the typical ${ }^{1} \mathrm{H}$ NMR spectrum of such a product, whose main impurities are constituted by fatty acids, acetic acid and $\mathrm{NaCl}$, but small fractions of additional compounds derived from side reactions can also pollute the system. We must highlight the following resonances belonging to various types of impurities, whereas all other peaks are directly related (not commented here) to the acidic form of sophorolipids:

- $\delta=0.92 \mathrm{ppm}$, typical of a $\mathrm{CH}_{3}$ group in an aliphatic chain, here attributed to residual fatty acids. This impurity is easy to recognize and distinguish from SL because the chemical shift corresponding to same group in SL is slightly unshielded.

- $\delta=1.96 \mathrm{ppm}$, typical of the $\mathrm{CH}_{3}$ group of free acetic acid, derived from acetate in the raw sophorolipids. This resonance is easy to attribute because of its sharpness.

- $\quad 6.30<\delta<5.70$, unknown impurities $\mathrm{N}^{\circ} 1$, derived from overheating issues 
- $\delta=8.51 \mathrm{ppm}$, unknown impurity $\mathrm{N}^{\circ} 2$, derived from overheating issues

The 9-5 ppm portion of the spectrum belonging to the raw mixture before hydrolysis is shown in the same figure for comparison, indicating the absence of any signal in this region. These resonances are the typical consequence of a poorly controlled hydrolysis reaction and are presented here on purpose, mainly for didactical purposes. The nature and way to remove these impurities, which are responsible for the dark brown/black colour of the mixture, will be discussed later and can be avoided by simply keeping the reaction temperature below $90^{\circ} \mathrm{C}$, as commented before.

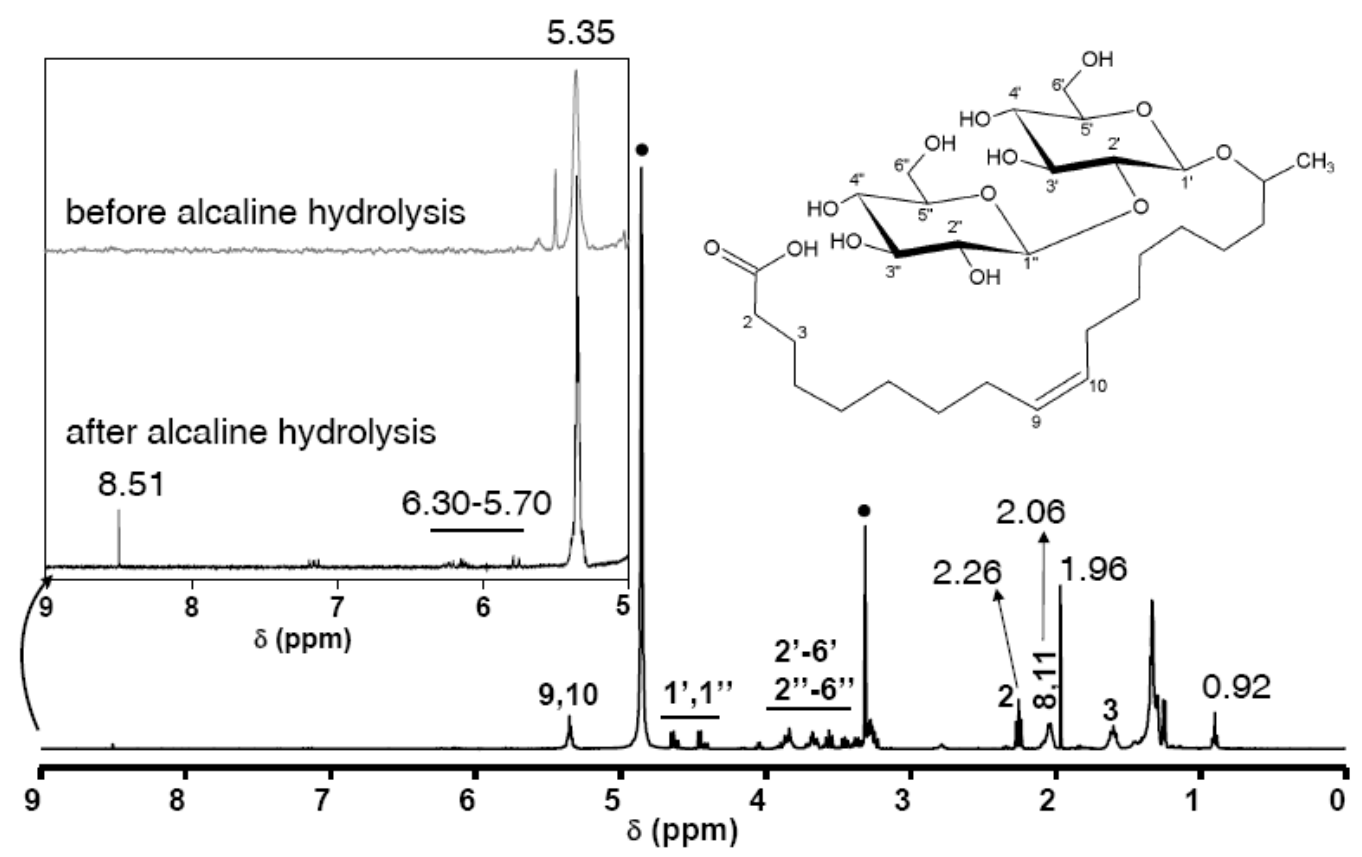

Figure $2-{ }^{1} \mathrm{H}$ NMR spectrum of the raw compound after alcaline hydrolysis. The 9-5 ppm region is specifically highlighted to show the effect of hydrolysis on the purity compound mixture before and after hydrolysis. Full black circle indicate the solvent resonances.

Method 1: Classic pentanol extraction. The hexane washing combined with pentanol extraction, followed by silica column chromatography purification, was described before by several authors. ${ }^{5,19}$ Unfortunately, very little specific information is available on this procedure, which has more than one inconvenience. First of all, pentanol is a difficult solvent to remove under vacuum, an operation which can take from hours to days according to the quantities and the type of apparatus employed for the task. In fact, removal of pentanol using low heating $\left(\mathrm{T} \leq 45^{\circ} \mathrm{C}\right)$ can be done with a cooled serpentine refrigerated below $\mathrm{T}=6^{\circ} \mathrm{C}$ and vacuum pressure below 10 mbar. These operating conditions are probably common for organic-chemistry laboratories but they are definitely not generalizable for an average, non- 
organic related, environment. When removal is done in the absence of a cooled serpentine, one must work with a standard water supply, of which the temperature does not fall below 15$18{ }^{\circ} \mathrm{C}$. To achieve complete solvent removal one can either raise the bath temperature up to $60-70{ }^{\circ} \mathrm{C}$, thus inducing the risk of esterification between pentanol and the sophorolipid $\mathrm{COOH}$ group, or work under very low vacuum conditions (below 1 mbar) at room temperature, which requires the use of a liquid nitrogen trap to condense the pentanol vapours. Even if a combination of both approaches can provide good results, the whole approach is time-consuming followed by many safety issues (potential glassware explosion, use of cryogenic fluids, long extraction time, etc.).

The best adapted experimental details for this purification procedure are given here. The pentanol/water phase transfer can be done on the previously hydrolyzed mixture using 5 x 100 $\mathrm{mL}$ portions of pentanol in a separatory funnel. Once all fractions are collected, one should take care of removing exceeding water using a pipette or magnesium sulfate. As for pentanol extraction, one can set a $47^{\circ} \mathrm{C}$ water bath and a cooling system at $6^{\circ} \mathrm{C}$. Vacuum pressure should be slowly lowered from 80 mbar to 8-10 mbar to initially remove residual water, then acetic acid and finally pentanol over 2-4 hours. The residual product has the aspect of a viscous brown/dark brown (large impurities) oily phase or a pale yellow/grey dried powder (low impurities). The main impurities are residual fatty acids, structural water and eventual oxidized compounds, mentioned previously. The eventual brownish colour depends on watersoluble species; so, if water is carefully removed before vacuum extraction, these should not be recovered in the end. On the contrary, fatty acids can only be removed by alcane (generally, hexane) washing. This procedure can take place either before, as previously suggested, alkaline hydrolysis on the raw product or after pentanol extraction, once a powder is obtained, using Soxhlet extraction, as we actually suggest here. Soxhlet extraction works in a closed circuit, thus allows solvent economy and efficient fatty acid removal, as well as all other organic impurities. An additional advantage of Soxhlet extraction concerns the removal of residual pentanol, which can contaminate the system. This is shown in Figure 3. The spectrum before pentanol extraction refers to a hydrolized acidic SL product which was actually initially washed with hexane. Three types of impurities can be found: residual fatty acids $(\delta=0.92)$, acetic acid $(\delta=1.96)$ and the above mentioned spurious impurities between 9 and 5 ppm. Clearly, a hexane washing prior to alkaline hydrolysis is not enough to remove residual hydrophobic compounds. Upon pentanol extraction, only the resonances at $8.51 \mathrm{ppm}$ and $1.96 \mathrm{ppm}$ are filtered out, showing that the impurity at $8.51 \mathrm{ppm}$ is also related to a hydrophilic compound. At this stage, the peak at $\delta=0.92$ is still present. One should note that 
residual pentanol can also be observed at this resonance. Finally, Soxhlet extraction removes all hydrophobic compounds (fatty acids and eventual residual pentanol) marking the spectrum at $\delta=0.92$ but also those impurities resonating between $6.30 \mathrm{ppm}$ and $5.70 \mathrm{ppm}$.

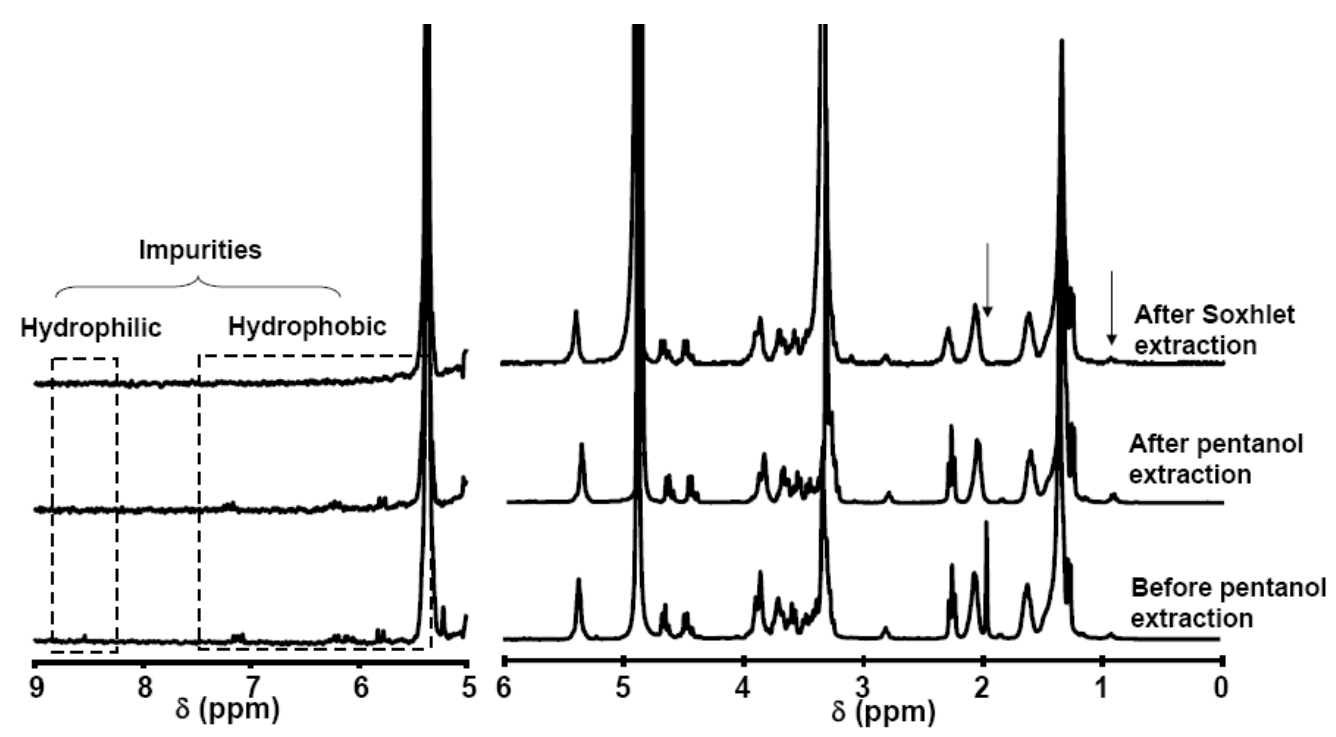

Figure $3-{ }^{1} \mathrm{H}$ NMR spectra showing the effects of the pentanol extraction process on the hydrolyzed sophorolipid. The top spectrum shows the effect of Soxhlet extraction on the pentanol-washed compound. The arrows indicate the impurities related to acetic acid and fatty acids.

Eventually, the positive point of using this route is the possibility to treat fairly large amounts of matter (here $20 \mathrm{~g}$ but this can definitely be increased) with good yields (above 80 $\mathrm{w} \%$ with respect to the initial mass of raw sophorolipids). In terms of final purity, the result is more than satisfactory. All hydrophilic compounds (acetic acid, hydrophilic impurities) are efficiently removed, provided that bulk water is removed as well. The level of hydrophobic compounds can be safely kept below 5 mol\%, as estimated by ${ }^{1} \mathrm{H}$ NMR, especially if Soxhlet treatment is performed and if the final powder is heated under strong vacuum.

The main drawback for this route is the absolute need of a performing, well-designed, vacuum rotavapor with a closed cooling system and an efficient pump. Under these conditions, this approach is safely suggested.

Method 2: Modified pentanol extraction. To overcome the drawbacks mentioned just above, it is also possible to recover the acidic sophorolipid from pentanol by decreasing the polarity of the medium. This can be easily done by adding an equi-volumetric amount of a short chain alcane (pentane, hexane or heptane) to the pentanol medium. The mixture can then be stored at $-18{ }^{\circ} \mathrm{C}$ to induce precipitation of the acidic sophorolipids. The product is then recovered by a simple filtering step. The final product is particularly pure as fatty acids remain in solution 
but further purification, if needed, can be performed using the already-mentioned Soxhlet extraction process.

This procedure is extremely easy to perform as no specific instrument is needed and it provides a product of good purity, below the detection limit of ${ }^{1} \mathrm{H}$ NMR, as shown by the corresponding spectrum in Figure 4.

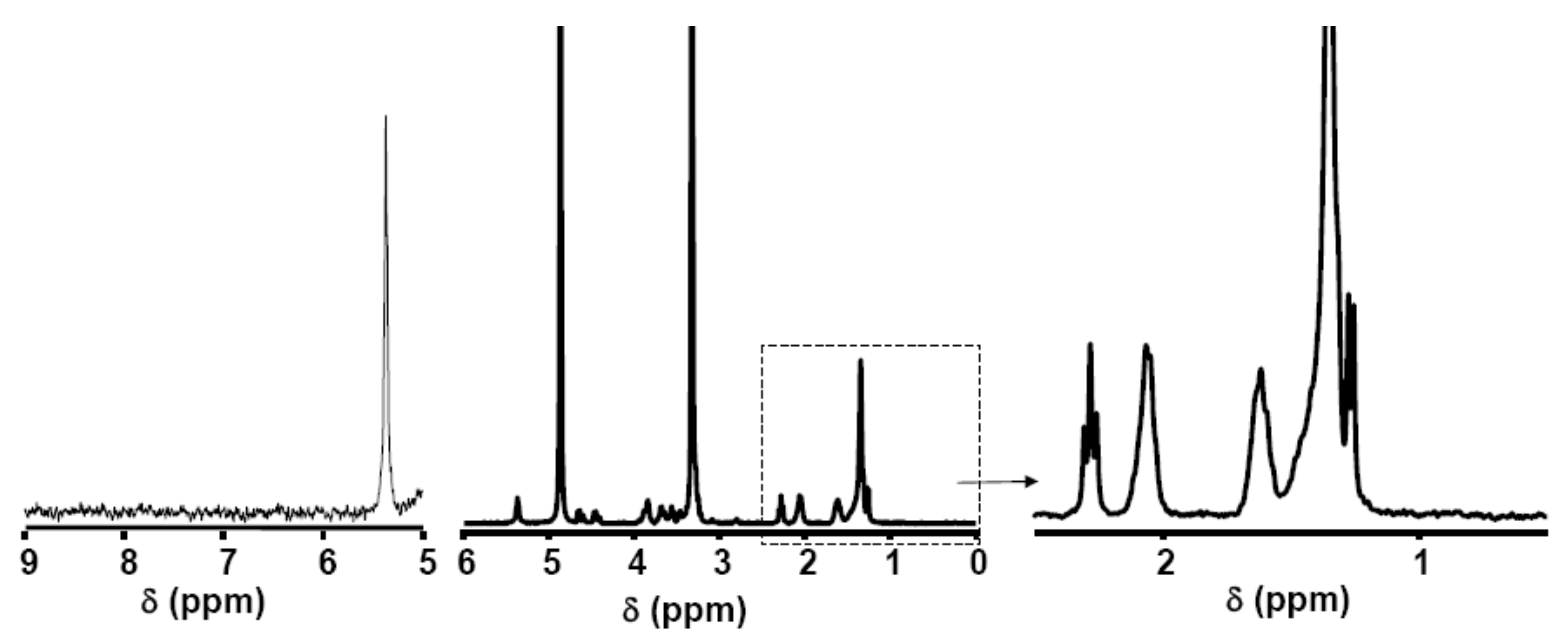

Figure $4-{ }^{1} \mathrm{H}$ NMR spectrum of the purified sophorolipid using the modified pentanol extraction method.

The 9-5 ppm and 0-2.5 ppm regions are highlighted to show the loss of all impurities. The typical spectrum before purification is given on Figure 3 (bottom spectrum).

Nevertheless, several drawbacks that may impact the overall time and energy consumption should also be pointed out. First of all, residence times at $-18^{\circ} \mathrm{C}$ are quite long $(>12 \mathrm{~h})$; secondly, extraction should be repeated several times (here, we went up to three times) to increase the yield, which are below $80 \%$. Finally, it is of crucial importance to remove any bulk water before storing the solution. In fact, if this is not done, the actual precipitate is a water-rich sophorolipid precipitate that melts at room temperature.

Method 3: Double silica filtration. On the basis of silica column chromatography experience, we propose here an adapted, fast and easy way to recover acidic sophorolipids. Experiments performed on a column are difficult to carry for large amounts (tens of grams) of compound. We have found large loss in matter and relative purification efficiency. For this reason, we adapted the column principle to work in a classical beaker, much easier to manipulate. As discussed previously, the hydrolyzed sophorolipid mixture contains both hydrophilic and hydrophobic impurities, in addition to sophorolipids themselves. Removal can then be done with a double silica phase extraction in the suited solvent mixture, where both hydrophilic silica gel 60 and hydrophobic C18-grafted silica are used at different times. The use of two 
different solid phases is preferred to a single one to always keep sophorolipids in the liquid phase, thus reducing adsorption/desorption phenomena which would consequently reduce the final yield. In the following, Figure 5, Figure 6 and Figure 7 and related discussion show the preliminary tests that are carried out on a given amount of hydrolyzed sophorolipids in the corresponding solvent to which the desired amounts of the adsorbant is added. At the end of this section, we show an all-in-one consecutive purification procedure (C18-modified silica followed by silica gel extraction) performed on larger amounts of the same hydrolyzed sophorolipid batch.

Figure 5 shows the typical HPLC chromatogram of hydrolized SL before silica filtration, showing three distinct regions corresponding to hydrophilic species (1.7 $\mathrm{min})$, sophorolipids (17.5-21.5 min) and fatty acids (34-40 min). ${ }^{20}$ This specific mixture containing three families of compounds with large differences in polarity is a good candidate for selective bulk silica adsorption.

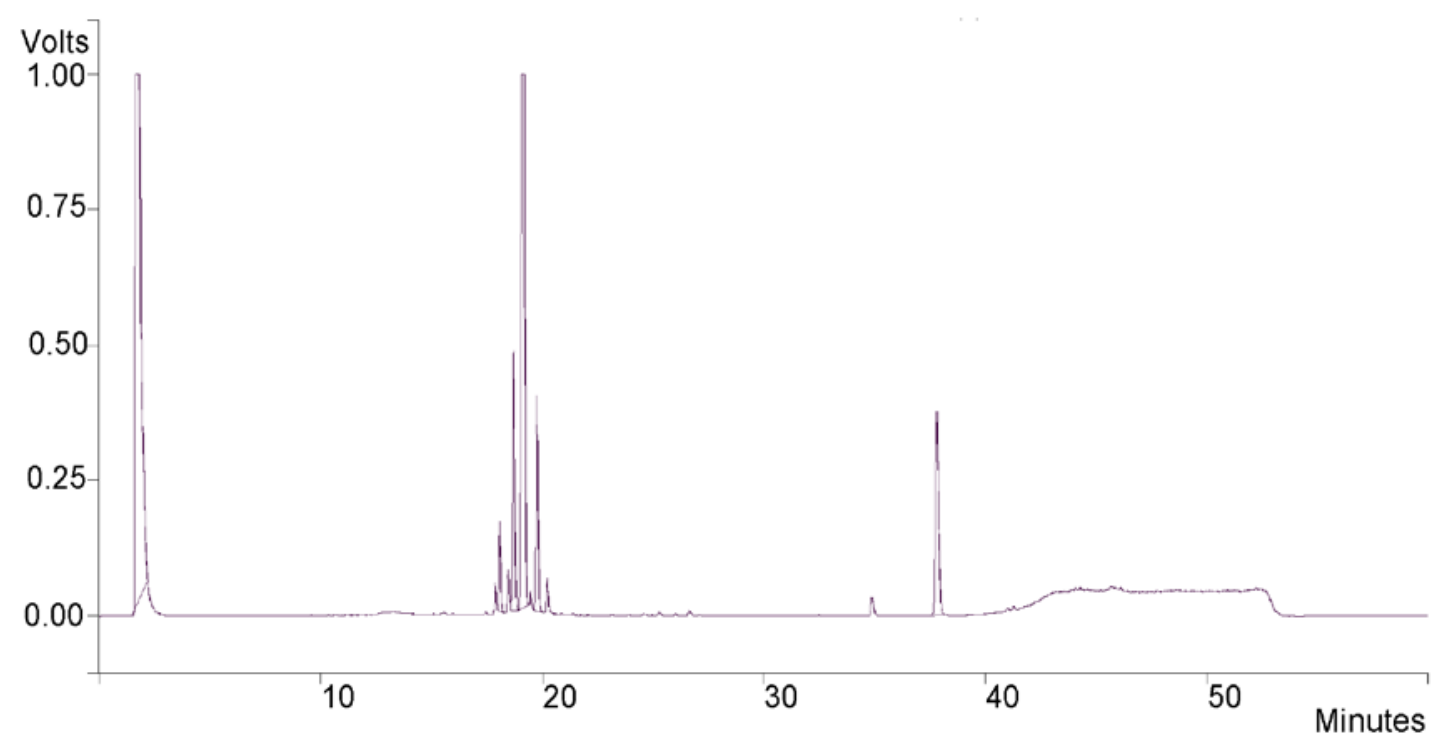

Figure 5 - Typical chromatogram of hydrolyzed SL. Peak attribution is done in the text.

The purification tests of hydrolyzed SL sample on bulk silica gel to remove hydrophilic impurities is shown on Figure 6, where a $\mathrm{CH}_{2} \mathrm{Cl}_{2} / \mathrm{CH}_{3} \mathrm{OH}=70 / 30$ solvent mixture is used. Sophorolipid concentration in the solvent is $50 \mathrm{mg} / \mathrm{mL}$; to this, various amounts of silica gel have been added. Solution is stirred for 10 minutes, left at rest and analyzed both using ${ }^{1} \mathrm{H}$ NMR and HPLC. NMR spectra (Figure 6) show that both the intensity of the resonance at $1.96 \mathrm{ppm}$ and $8.95 \mathrm{ppm}$ decrease with the amount of $\mathrm{SiO}_{2}$ in the solutions. In particular, the resonance at $8.95 \mathrm{ppm}$, referred to a minor amount of an unidentified species, completely disappear while the resonance at $1.96 \mathrm{ppm}$, attributed to acetic acid, is still present in very low amount. The quantification will be discussed later. HPLC experiments in Figure 6 confirm 
these results as the contribution of the hydrophilic compounds eluting at 1.7 min indeed diminishes.

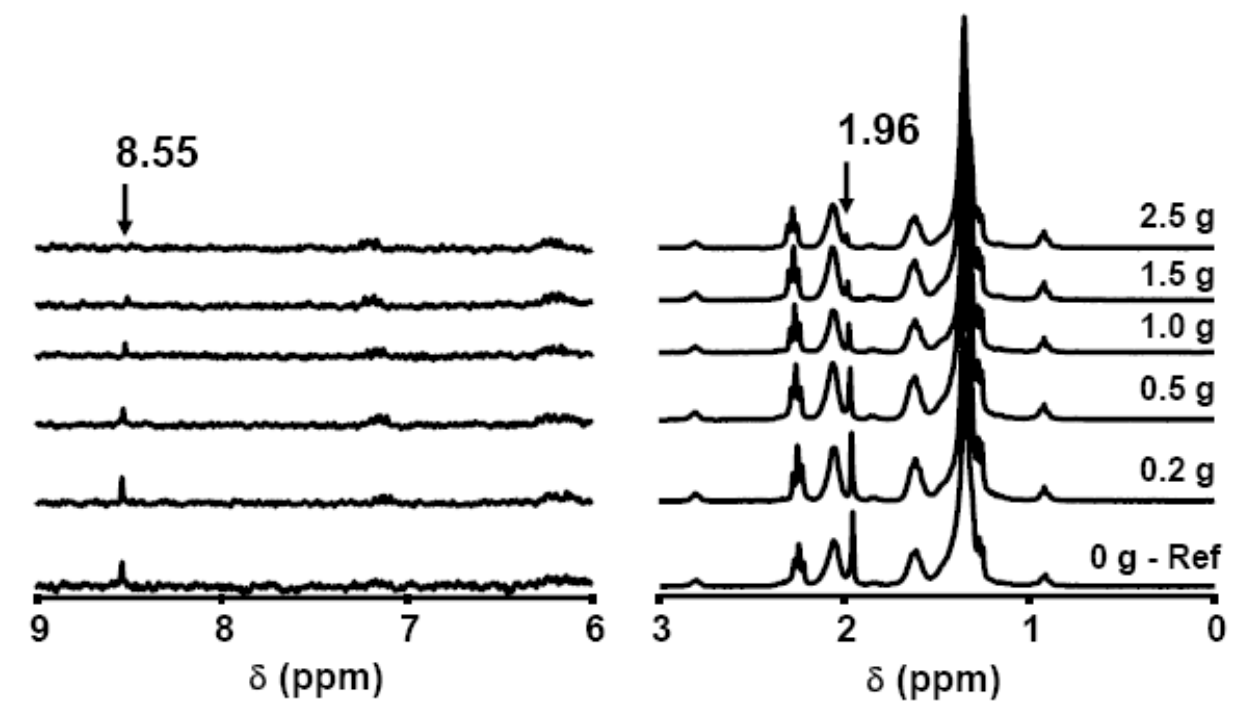

a)

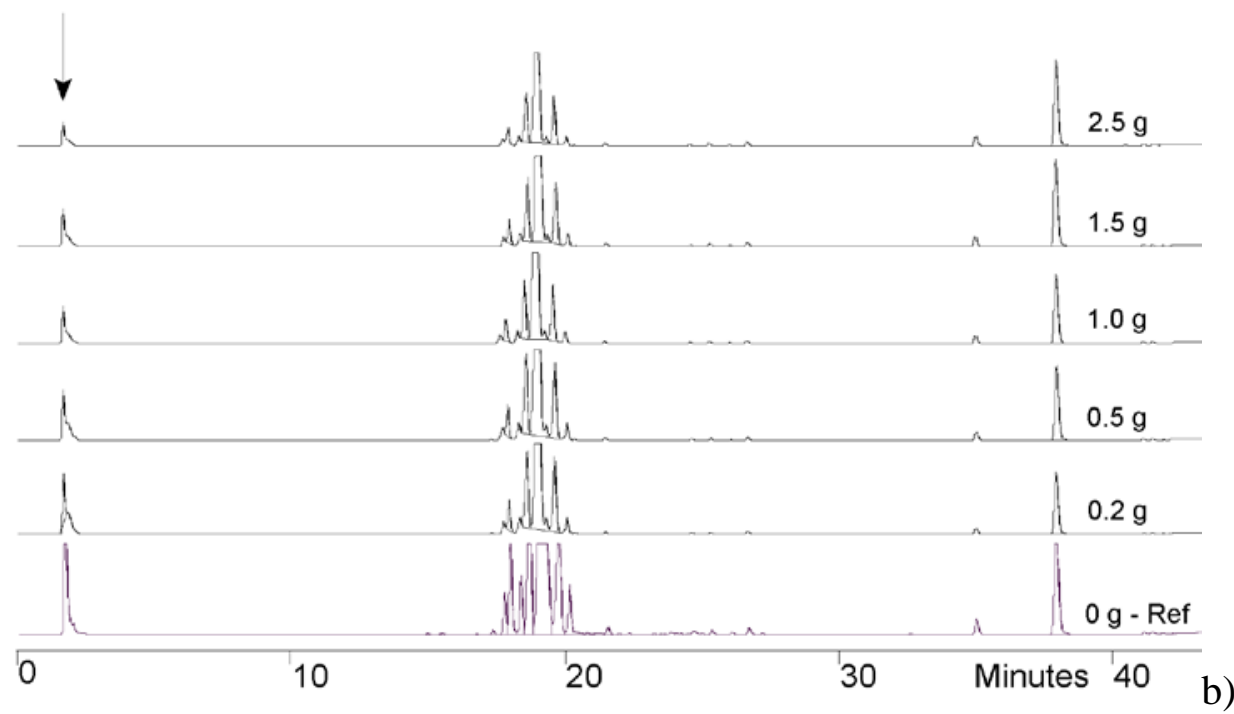

Figure 6 - a) 1H NMR and b) HPLC data showing the effect of increasing amounts of silica gel (given on the figure) on the adsorption of the hydrophilic impurities from the hydrolyzed form of sophorolipids. Sophorolipid concentration is $50 \mathrm{mg} / \mathrm{mL}$, while the volume of the solution is $10 \mathrm{~mL}$.

Interestingly, one can clearly see that this procedure has no effect at all on filtering out the hydrophobic species, whose NMR signals at $0.92 \mathrm{ppm}$ and 6.30-5.70 ppm and HPLC retention times between 34 and $40 \mathrm{~min}$, refer to. On the contrary, the elimination of these compounds can be efficiently done using a bulk amounts of C18-modified silica powder. Sample preparation is similar for the previous experiment except for the solvent mixture. In this case one can work in $\mathrm{H}_{2} \mathrm{O}$ /acetonitrile $=90 / 10$. NMR experiments (Figure 7) indicate that small amounts of modified powder is necessary to remove residual fatty acids and 
hydrophobic impurities, whereas hydrophilic compounds are left in the solution and are not retained, as shown by the persistence of the peak at $8.55 \mathrm{ppm}$. This is even clearer in the HPLC experiments on Figure 7b where the hydrophilic material eluting in the beginning of the analysis remains untouched while the free fatty acids detected 34 and 40 minutes are removed by adding $1.0 \mathrm{~g}$ of C18-modified silica powder.

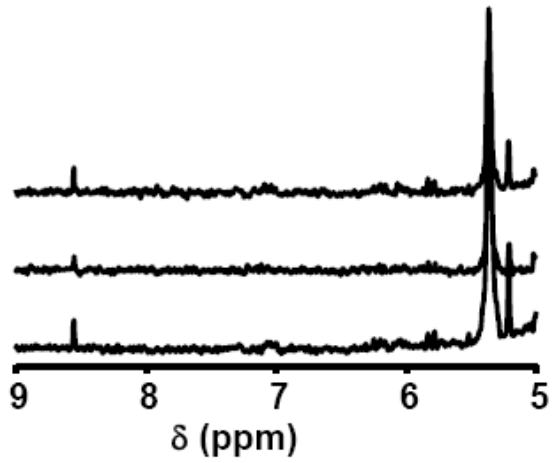

$\delta(\mathrm{ppm})$

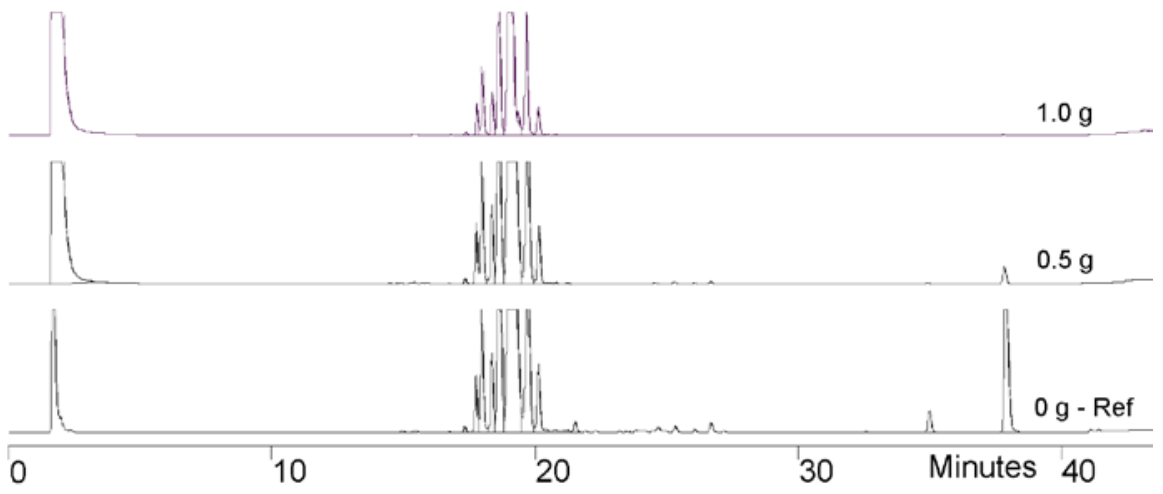

a)

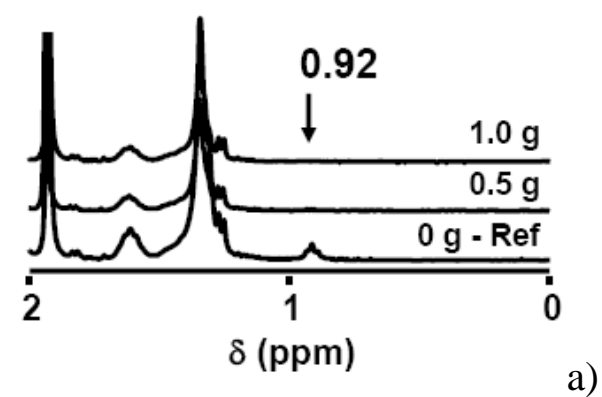

b)

Figure 7 - a) ${ }^{1} \mathrm{H}$ NMR and b) HPLC data showing the effect of increasing amounts of C18-modified silica (given on the figure) on the adsorption of the hydrophilic impurities from the hydrolyzed form of sophorolipids. Sophorolipid concentration is about $90 \mathrm{mg} / \mathrm{mL}$, while the volume of the solution is $10 \mathrm{~mL}$.

These tests indicate the good efficiency of the double silica-based treatment. Even if the advantage of changing solvents and substrates is to always keep sophorolipids in the liquid phase so to limit their adsorption/desorption, we still do experience a loss of SL-COOH in the process, as testified by the HPLC data (Figure 6b and Figure $7 \mathrm{~b}$ ), where the SL signal in the 17.5-21.5 min region is also affected by the filtering process.

To quantify this procedure in terms of both recovered amount of sophorolipids and residual impurities, we have treated the same initial sophorolipid batch in a consecutive way starting from the hydrolyzed form of the as-provided compound. Since this is dissolved in an aqueous medium and to limit the number of overall steps, we prepared a $100 \mathrm{mg} / \mathrm{mL}$ solution from the hydrolyzed batch itself, where the solvent is constituted by a water/acetonitrile 90/10 mixture 
and used an overall volume of $100 \mathrm{~mL}$, to which were added $5 \mathrm{~g}$ of $\mathrm{C} 18-\mathrm{SiO}_{2}$. This was stirred at room temperature and after about $30 \mathrm{~min}$ the silica phase was removed by filtration. The aqueous liquid phase was removed under reduced pressure, being extremely careful to remove water. The solute was redissolved in about $400 \mathrm{~mL}$ of $\mathrm{CH}_{2} \mathrm{Cl}_{2} / \mathrm{CH}_{3} \mathrm{OH}-70 / 30$, to which about $100 \mathrm{~g}$ of silica gel was added and filtered after the adsorption of the hydrophilic impurities. This is quite clear as for the most polluted solutions, which colours are brownish, one can clearly see an increase of transparency upon filtration, the darkest compounds being adsorbed on the solid phase. We found this part being the most critical step for this process, given the large amount of silica employed. It is advisable to rinse the collected solid phase at least once or twice with an equivalent amount of the solvent mixture. Finally, all solvent fractions are grouped together and evaporated under reduced pressure.

This method has the advantage to provide an easy and fast way to recover an acidic sophorolipid compound but two main drawbacks must be outlined: the lower yield (about 50/60 \%) and higher impurity with respect to the pentanol routes. The first point depends on the large amounts of silica gel employed, which retain part of the sophorolipid in solution. The second point depends on the efficiency in terms of impurity adsorption for large amount of matter. Both of them could be improved by a better selection of the solvent mixtures, for instance. The typical spectra obtained for the compound before and after treatment are shown in Figure 8. One can observe the drastic reduction in both the peak related to the hydrophilic and hydrophobic impurities, even if both are still observed and whose amount is estimated to be below $10 \mathrm{~mol} \%$. 


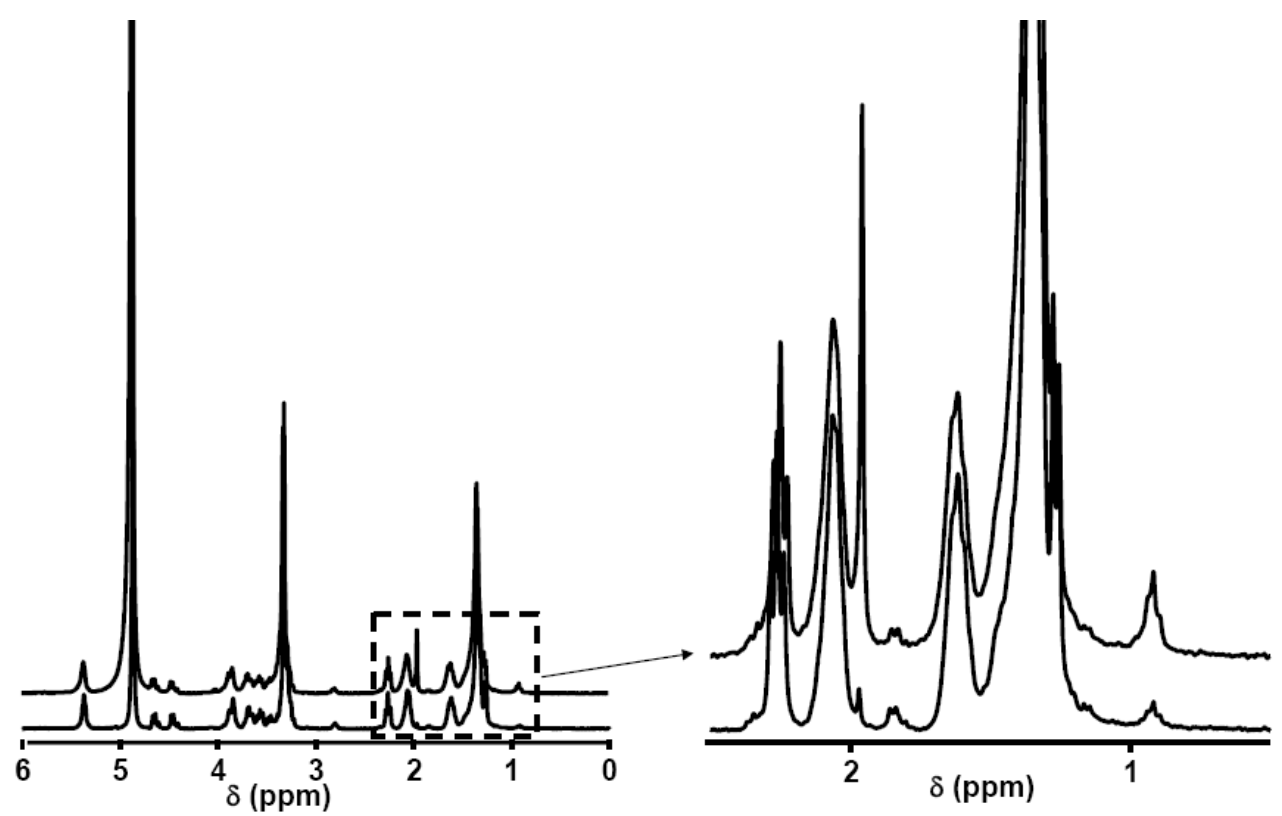

Figure 8 - ${ }^{1} \mathrm{H}$ NMR spectra of the SL-COOH (top spectrum) consecutively purified with silica gel and C18-modified silica (bottom spectrum). Spectra are normalized.

In the following table we highlight the advantages and disadvantages for each method presented above. All in all, the pentanol route is quite robust and it provides a rather pure, readily desalted, product. Nevertheless, we do not recommend using the pentanol extraction under reduced pressure in the absence of a well-equipped vacuum extraction system. The most practical and best way in terms of purity is undoubtedly the precipitation in a cold pentanol/alcane mixture, but this is a long way (several days) if one wants to obtain the highest yield possible. Finally, the double silica filtration is an interesting, very fast, method for immediate use, even if lower yields and residual impurities are higher with respect to the pentanol routes. For possible scale-up, further work must be done to develop these processes and reduce all inconvenients, thus maximizing yields and minimizing impurities. For instance, in the double silica extraction, one could try to adjust the polarity of the solvents and use optimized amounts of both silica gel and inversed silica phase. In the modified pentanol route, on the contrary, one could use a more efficient solvent combined to pentanol to reduce the precipitation times.

\begin{tabular}{|c|c|c|c|c|}
\hline Method & Yield* & Impurities+ & Advantages & Disadvantages \\
\hline $\begin{array}{c}\text { Pentanol } \\
\text { extraction }\end{array}$ & $\leq 90 \%$ & $\begin{array}{c}<5 \mathrm{~mol} \% \\
(\delta=0.92 \mathrm{ppm})\end{array}$ & Quantitative & $\begin{array}{c}\text { Need for improved vacuum extraction } \\
\text { equipment for solvent evaporation. } \\
\text { Possible esterification reactions at bath } \\
\text { temperatures above } 60^{\circ} \mathrm{C}-70^{\circ} \mathrm{C} .\end{array}$ \\
\hline $\begin{array}{c}\text { Modified } \\
\text { pentanol } \\
\text { extraction }\end{array}$ & $\leq 80 \%$ & None & $\begin{array}{c}\text { Fairly } \\
\text { quantitative. } \\
\text { High purity. }\end{array}$ & Long \\
\hline
\end{tabular}




\begin{tabular}{|c|c|c|c|c|}
\hline & & & $\begin{array}{l}\text { No need for } \\
\text { vaccum } \\
\text { extraction. }\end{array}$ & \\
\hline $\begin{array}{l}\text { Double silica } \\
\text { filtration }\end{array}$ & $\leq 60 \%$ & $\begin{array}{c}<2 \mathrm{~mol} \% \\
(\delta=1.96) \\
<6 \mathrm{~mol} \% \\
(\delta=0.92 \mathrm{ppm})\end{array}$ & $\begin{array}{c}\text { Fast. } \\
\text { Easy vacuum } \\
\text { extraction. }\end{array}$ & $\begin{array}{c}\text { Average yield } \\
\text { Slightly larger amounts of impurities }\end{array}$ \\
\hline
\end{tabular}

*= amount (in grams) of recovered sophorolipid powder with respect to the effective sophorolipid amount used. + Initial impurities: acetic acid: 20 mol\%; fatty acid: $12 \mathrm{~mol} \%$. Calculation of impurities is done using ${ }^{1} \mathrm{H}$ NMR resonances $\delta=1.96\left(\mathrm{CH}_{3} \mathrm{COOH}\right)$ and $\delta=0.92 \mathrm{ppm}\left(\mathrm{R}_{-}-\mathrm{CH}_{2}-\mathrm{CH}_{3}\right)$ with respect to the given SL peak at $\delta=2.06$ $\operatorname{ppm}\left(\mathrm{R}-\underline{\mathrm{C}}_{2}-\mathrm{CH}_{2}-\mathrm{COOH}\right)$.

\section{Conclusion}

This work has shown the possibilities offered by two alternative routes in the extraction process of acidic sophorolipids in a water solution after the base-catalyzed hydrolysis of the lactonic form in a commercial formulation. We initially detailed the classical pentanol extraction route, which consists of a hexane washing (to remove residual fatty acids) previous to solvent/solvent extraction at room temperature followed by the pentanol vacuum removal. This process, almost quantitative and providing a good quality product, has several limitations and drawbacks to treat large amounts of matter (tens of grams): 1) manipulation of large amounts of solvents (pentanol, hexane); 2) need for a specific vacuum extraction setup for rapid and effective pentanol removal. In particular, the second point is the most limiting one to operate the extraction correctly and in the absence of a specific apparatus with a closed refrigerating system, the extraction can be extremely long and the product has good chances to be polluted with residual pentanol. Furthermore, esterification between pentanol and the $\mathrm{COOH}$ group of $\mathrm{SL}$ is possible if one rises the heating temperature to $60^{\circ} \mathrm{C}-70^{\circ} \mathrm{C}$ for several hours. To overcome these drawbacks, we have proposed two alternative routes. In the first one, one can perform a cold extraction by adding an alcane in a 1:1 v:v proportion to the SLcontaining pentanol solution. This method is very effective in terms of final purity as only acidic sophorolipids precipitate while all other hydrophobic impurities (e.g., fatty acids) remain in solution. This way should also avoid the need to pre-treat the raw sophorolipid mixture with hexane washing. Several problems are inherent to this approach: its length (up to several days for a quantitative extraction) and the presence of residual water bound to sophorolipids, which should be carefully removed before product filtration. Further development in terms of best co-solvent and careful bulk water removal could greatly help to overcome these points. In summary, this method is by far more practical and safer than the first one and it can be employed in any, non organic-chemistry specialized, environment. 
Nevertheless, in the quest for a fast production, one can try the third approach that we propose, consisting in a double solid/liquid extraction on a C18-modified silica powder and normal silica gel. This method, adapted from column chromatography, can be developed here because of the little heterogeneity of the raw product after alkaline hydrolysis: hydrophilic compounds (e.g., acetic acid, minor impurities), hydrophobic molecules (fatty acids, if not removed in advance) and acidic sophorolipids of average hydrophilicity. This is a fast track approach with several drawbacks, that can probably be overcome with further process improvement. Due to undesired solid-phase adsorption of sophorolipids, this is by far the least quantitative method described so far and in spite of the designed choice of solid/liquid duo that allows to always keep sophorolipids in the liquid phase. So far, this method is also the least effective in terms of impurities removal (especially the most hydrophilic ones), even if the overall amount can be as low as $6 \%$. On the contrary, this method employs volatile solvents easy to remove with a standard rotavapor apparatus. Finally, we also identify several points that one should consider for a successful extraction, such as the limitation in the hydrolysis temperature, which if too high it promotes the formation of hydrophilic impurities, the use of Soxhlet extraction to remove fatty acids at the end rather than at the beginning of the process, thus limiting the amount of employed hexane, the careful removal of bulk water from the pentanol mixture, use of low temperatures when working with pentanol to avoid esterification, etc...

In summary, we have shown multiple ways that one can follow to recover acidic sophorolipids, proposing the positive and negative aspects for each one and indicating how these methods could be developed further to overcome the drawback and, eventually, to end up with an up-scalable, highly performing, process.

\section{Acknowledgements}

The research leading to these results has received funding from the European Community's Seventh Framework Programme (FP7/2007-2013) under Grant Agreement $n^{\circ}$ Biosurfing/289219 and the French state funds managed by the ANR within the Investissement d'Avenir programme under the reference ANR-11-IDEX-0004-02 (Labex MATISSE). We kindly acknowledge Dr. Cyril Martini and Dr. François Ribot (Laboratoire de Chimie de la Matière Condensée de Paris, Collège d France/UPMC/CNRS, Paris, France) and Dr. Philippe Simon (Chimie des Processus Biologiques, Collège de France, Paris, France) for helpful discussions. Stijn Verweire (InBio, Ghent University, Ghent, Belgium) is acknowledged for technical assistance on HPLC. 


\section{References}

${ }^{1}$ Kjellin, M.; Johansson, I. Surfactants from Renewable Resources, John Wiley \& Sons, Ltd: West Sussex, 2010

${ }^{2}$ von Rybinski, W.; Hill, K. Alkyl Polyglycosides-Properties and Applications of a new Class of Surfactants Angew. Chem. Int. Ed. 1998, 37, 1328-1345

${ }^{3}$ Stubenrauch, C. Sugar Surfactants - Aggregation, Interfacial, and Adsorption Phenomena Curr. Op. Coll. Interf. Sci. 2001, 6, 160-170

${ }^{4}$ Hoffmann, B.; Platz, G. Phase and Aggregation Behaviour of Alkylglycosides Curr. Op. Coll. Interf. Sci. 2001, 6, 171-177

${ }^{5}$ Rau, U.; Hammen, S.; Heckmann, R.; Wray, V.; Lang, S. Sophorolipids: a Source for Novel Compounds Ind. Crops Prod. 2001, 13, 85-92

${ }^{6}$ Tulloch, A. P.; Hill, A.; Spencer, J. F. T. Structure and Reactions of Lactonic and Acidic Sophorosides of 17Hydroxyoctadecanoic Acid Canad. J. Chem. 1968, 46, 3337-3351

${ }^{7}$ Asmer, H.-J. ; Lang, S.; Wagner, F.; Wray, V. Microbial Production, Structure Elucidation and Bioconversion of Sophorose Lipids. J. Am. Oil. Chem. Soc. 1988, 65, 1460-1466

${ }^{8}$ Zhou, S.; Xu, C.; Wang, J.; Gao, W.; Akhverdiyeva, R.; Shah, V.; Gross, R. Supramolecular Assemblies of a Naturally Derived Sophorolipid Langmuir 2004, 20, 7926-7932

${ }^{9}$ Develter, D. W. G.; Fleurackers, S. J. J. Surfactants from Renewable Resources, John Wiley \& Sons, Ltd: West Sussex, 2010; pp. 213-238

${ }^{10}$ Maingault, M. Use of Sophorolipids and Cosmetic and Dermatological Compositions, WO/1995/034282A

${ }^{11}$ Van Bogaert, I. N. A.; Saerens, K.; De Muynck, C.; Develter, D.; Soetaert, W.; Vandamme, E. J. Microbial Production and Application of Sophorolipids Appl. Microbiol. Biotechnol. 2007, 76, 23-34

12 Shete, A. M.; Wadhawa, G.; Banat, I. M.; Chopade, B. A. Mapping of Patents on Bioemulsifier and Biosurfactant: a Review J. Sci. Ind. Res. 2006, 65, 91-11

${ }^{13}$ Fu, S. L.; Wallner, S. R.; Bowne, W. B.; Hagler, M. D.; Zenilman, M. E.; Gross, R.; Bluth, M. H. Sophorolipids and Their Derivatives are Lethal Against Human Pancreatic Cancer Cells J. Surg. Res. 2008, 148, 77-82

${ }^{14}$ Baccile, N.; Nassif, N.; Malfatti, L.; Van Bogaert, I. N. A.; Soetaert, W.; Pehau-Arnaudet, G.; Babonneau, F. Sophorolipids: a Yeast-Derived Glycolipid as Greener Structure Directing Agents for Self-Assembled Nanomaterials Green Chem. 2010, 12, 1564-1567

${ }^{15}$ N. Baccile, F. Babonneau, J. Jestin, G. Pehau-Arnaudet, I. Van Bogaert, ACS Nano, 2012, 6, 4763-4776

${ }^{16}$ N. Baccile, J. S. Pedersen, G. Pehau-Arnaudet, I. Van Bogaert, Soft Matter, 2013, DOI:10.1039/C3SM50160D

${ }^{17}$ P. Dhasaiyan, A. Banerjee, N. Visaveliya, B. L. V. Prasad, Chem. Asian J., 2013, 8, 369-372

18 Saerens, K.M.J., Saey, L. and Soetaert, W. One-step production of unacetylated sophorolipids by an acetyltransferase negative Candida bombicola. Biotechnol Bioeng, 2011, 108, 2923-2931

${ }^{19}$ U. Rau, R. Heckmann, V. Wray, S. Lang, Biotechnol. Lett., 1999, 21, 973-977

${ }^{20}$ Van Bogaert, I.N. A., Fleurackers, S., Van Kerrebroeck, S., Develter, D. \& Soetaert, W. Production of new-tonature sophorolipids by cultivating the yeast Candida bombicola on unconventional hydrophobic substrates. Biotechnol. Bioengin., 2011, 108, 734-741. 